\title{
THE EFFECT OF RANDOMNESS ON THE STABILITY OF DEEP WATER SURFACE GRAVITY WAVES IN THE PRESENCE OF A THIN THERMOCLINE
}

\author{
SUDEBI BHATTACHARYYA ${ }^{1}$ and K. P. DAS ${ }^{2}$
}

(Received 15 November 1996; revised 22 July 1997)

\begin{abstract}
The effect of randomness on the stability of deep water surface gravity waves in the presence of a thin thermocline is studied. A previously derived fourth order nonlinear evolution equation is used to find a spectral transport equation for a narrow band of surface gravity wave trains. This equation is used to study the stability of an initially homogeneous Lorentz shape of spectrum to small long wave-length perturbations for a range of spectral widths. The growth rate of the instability is found to decrease with the increase of spectral widths. It is found that the fourth order term in the evolution equation produces a decrease in the growth rate of the instability. There is stability if the spectral width exceeds a certain critical value. For a vanishing bandwidth the deterministic growth rate of the instability is recovered. Graphs have been plotted showing the variations of the growth rate of the instability against the wavenumber of the perturbation for some different values of spectral width, thermocline depth, angle of perturbation and wave steepness.
\end{abstract}

\section{Introduction}

A fourth order nonlinear evolution equation for a surface gravity wave packet in the presence of a thin thermocline has been obtained by the present authors in a previous paper [2]. By the use of this equation a deterministic growth rate of the instability has also been obtained in that paper. The reason for using a fourth order evolution equation is that according to Dysthe [4] a fourth order evolution equation gives results consistent with the exact results of Longuet-Higgins [5], [6] for waves of wave steepness up to 0.25 .

The effect of randomness on the stability of surface gravity waves has been investigated by Alber [1] and Crawford, Saffman and Yuen [3], and they started respectively

\footnotetext{
${ }^{1}$ Department of Mathematics, Scottish Church College, 1 \& 3, Urquhart Square, Calcutta-700006, India. ${ }^{2}$ Department of Applied Mathematics, University of Calcutta, 92, Acharya Prafulla Chandra Road, Calcutta-700009, India

(C) Australian Mathematical Society, 1998, Serial-fee code 0334-2700/98
} 
from the third order nonlinear evolution equation and Zakharov's integral equation [7]. A similar study is made in the present paper for surface gravity waves in the presence of a thin thermocline and we start from a fourth order nonlinear evolution equation obtained in our previous paper [2]. A spectral transport equation is derived from this equation. This spectral transport equation is used to study the stability of an initially homogeneous more realistic Lorentz shape of spectrum considered by Crawford, Saffman and Yuen [6]. A dispersion relation is obtained for oblique plane wave perturbation, from which an expression for the growth rate of instability is determined. This expression shows that a fourth order term in the nonlinear evolution equation, which is responsible for the wave-induced mean flow, has a contribution in the growth rate of instability and this produces a decrease in the growth rate of the instability. The growth rate of the instability decreases with the increase of spectral width, and the instability vanishes (that is, there is stability) if the spectral width increases beyond a certain critical value. By setting the spectral band width equal to zero, we recover the deterministic growth rate of the instability. Graphs are plotted showing the variations of growth rate against the wavenumber of the perturbation for some different values of spectral width, angle of perturbation and wave steepness. Graphs are also plotted showing stable-unstable regions in $\bar{l} \bar{\sigma}$-space, where $\bar{l}$ and $\bar{\sigma}$ are respectively the effective wave number and effective band-width parameter.

\section{The nonlinear evolution equation}

The wave height $\zeta(x, y, t)$ of a weakly nonlinear progressive wave above the undisturbed free surface of water in the presence of a thin thermocline situated at a depth $d$ below the undisturbed free surface can be written in the form

$$
\begin{aligned}
\zeta= & \left\{\zeta_{1}(X, Y, T) \exp [i(k x-\omega t)]+\zeta_{1}^{*}(X, Y, T) \exp [-i(k x-\omega t)]\right\} \\
& +(\text { zeroth and higher harmonic terms }),
\end{aligned}
$$

where

$$
X=\epsilon\left(x-c_{g} t\right), \quad Y=\epsilon y, \quad T=\epsilon^{2} t,
$$

$c_{g}=d \omega / d k$ being the group velocity of the wave, $\epsilon$ being a small parameter indicating the weakness of the amplitude $\zeta_{1}, \omega$ and $k$ satisfying the linear dispersion relation

$$
\omega^{2}=g k
$$

The evolution of the complex amplitude $\zeta_{1}$ is governed by the following fourth order nonlinear evolution equation, which has recently been derived by the present 
authors [2]

$$
i \frac{\partial \zeta}{\partial \tau}+\beta_{1} \frac{\partial^{2} \zeta}{\partial \xi^{2}}+i \beta_{2} \frac{\partial^{3} \zeta}{\partial \xi^{3}}=\Lambda_{1} \zeta^{2} \zeta^{*}+i \Lambda_{2} \zeta \zeta^{*} \frac{\partial \zeta}{\partial \xi}+i \Lambda_{3} \zeta^{2} \frac{\partial \zeta^{*}}{\partial \xi}+\Lambda_{4} \zeta \mathrm{H} \frac{\partial}{\partial \xi}\left(\zeta \zeta^{*}\right)
$$

where $\zeta=\epsilon \zeta_{11}+\epsilon^{2} \zeta_{12}, \zeta_{11}$ and $\zeta_{12}$ being the first two terms in the expansion of $\zeta_{1}$ in powers of $\epsilon$ and where it has been assumed that the space variation of the complex amplitude $\zeta$ takes place along a line making an arbitrary fixed angle $\theta$ with the direction of propagation of the wave and $\xi$ is the co-ordinate along this line. The quantities wave amplitude $\zeta$, space co-ordinate $\xi$ and time $\tau$ have been made dimensionless by the factors $k, k$ and $\omega$ respectively. The following are the coefficients of in (4):

$$
\begin{gathered}
\beta_{1}=-\frac{1}{8}\left(\cos ^{2} \theta-2 \sin ^{2} \theta\right), \quad \beta_{2}=-\frac{1}{16}\left(\cos ^{3} \theta-6 \cos \theta \sin ^{2} \theta\right), \\
\Lambda_{1}=2+\frac{8 \gamma \cos ^{2} \theta}{\cos ^{2} \theta-4 \gamma k d}, \Lambda_{2}=-6 \cos \theta, \Lambda_{3}=-\cos \theta, \Lambda_{4}=\frac{2 \cos ^{4} \theta}{\cos ^{2} \theta-4 \gamma k d} .
\end{gathered}
$$

The Hilbert transform operator $\mathrm{H}$ is given by

$$
\mathrm{H} \psi=\frac{1}{\pi} P \int_{-\infty}^{\infty} \frac{\psi\left(\xi^{\prime}\right)}{\xi^{\prime}-\xi} d \xi^{\prime}
$$

and $\gamma$ is given by $\gamma=\delta \rho / \rho, \delta \rho$ being the density increase through the thermocline.

As the coefficients $\Lambda_{1}$ and $\Lambda_{4}$ contain the factor $\cos ^{2} \theta-4 \gamma k d$ in their denominators, the evolution equation (4) does not remain valid when $\cos ^{2} \theta=4 \gamma k d$, which is the resonance condition. Moreover since the evolution equation (4) has been derived on the assumption that $k d$ is finite, the results for $k d \rightarrow \infty$ cannot be obtained from the evolution equation (4).

\section{The equation for the correlation function}

We assume that the complex amplitude $\zeta(\xi, \tau)$ is a random function of $\xi$. We now find an equation for the slow variation of the two point space correlation function,

$$
\rho\left(\xi_{1}, \xi_{2}, \tau\right)=\left\langle\zeta\left(\xi_{1}, \tau\right) \zeta^{*}\left(\xi_{2}, \tau\right)\right\rangle,
$$

where the angular bracket denotes ensemble average. To obtain this equation we adopt the method followed by Alber [1].

Equation (4) can be written as

$$
i \frac{\partial \zeta}{\partial \tau}+\beta_{1} \frac{\partial^{2} \zeta}{\partial \xi^{2}}+i \beta_{2} \frac{\partial^{3} \zeta}{\partial \xi^{3}}=\Gamma \zeta
$$


where

$$
\Gamma=\Lambda_{1} \zeta \zeta^{*}+i \Lambda_{2} \zeta^{*} \frac{\partial \zeta}{\partial \xi}+i \Lambda_{3} \zeta \frac{\partial \zeta^{*}}{\partial \xi}+\Lambda_{4} \mathrm{H} \frac{\partial}{\partial \xi}\left(\zeta \zeta^{*}\right)
$$

To obtain an equation for the slow variation of the two-point space correlation function $\rho$ defined by (7), the equation (8) at the point $\xi_{1}$ is multiplied by $\zeta^{*}\left(\xi_{2}, \tau\right)$. Next the complex conjugate of (8) at $\xi_{2}$ is multiplied by $\zeta\left(\xi_{1}, \tau\right)$. Finally subtracting the last equation from the former and then taking the ensemble average we get the equation

$$
\begin{aligned}
i \frac{\partial}{\partial \tau}\langle\zeta & \left.\left(\xi_{1}, \tau\right) \zeta^{*}\left(\xi_{2}, \tau\right)\right\rangle \\
& +\beta_{1}\left[\frac{\partial^{2}}{\partial \xi_{1}^{2}}\left\langle\zeta\left(\xi_{1}, \tau\right) \zeta^{*}\left(\xi_{2}, \tau\right)\right\rangle-\frac{\partial^{2}}{\partial \xi_{2}^{2}}\left\langle\zeta\left(\xi_{1}, \tau\right) \zeta^{*}\left(\xi_{2}, \tau\right)\right\rangle\right] \\
& +i \beta_{2}\left[\frac{\partial^{3}}{\partial \xi_{1}^{3}}\left\langle\zeta\left(\xi_{1}, \tau\right) \zeta^{*}\left(\xi_{2}, \tau\right)\right\rangle+\frac{\partial^{3}}{\partial \xi_{2}^{3}}\left\langle\zeta\left(\xi_{1}, \tau\right) \zeta^{*}\left(\xi_{2}, \tau\right)\right\rangle\right] \\
& =\left\langle\zeta\left(\xi_{1}, \tau\right) \zeta^{*}\left(\xi_{2}, \tau\right) \cdot\left[\Gamma\left(\xi_{1}, \tau\right)-\Gamma^{*}\left(\xi_{2}, \tau\right)\right]\right\rangle .
\end{aligned}
$$

Introducing average and spatial separation of coordinates $(X, r)$,

$$
X=\frac{1}{2}\left(\xi_{1}+\xi_{2}\right), \quad r=\xi_{1}-\xi_{2},
$$

(10) can be expressed as follows, where to evaluate fourth order correlation terms we assume that $\zeta(X, \tau)$ corresponds initially to a Gaussian random process and we further assume that the evolving random statistical amplitude field retains the same Gaussian statistical properties [1]. For Gaussian statistics the fourth order cumulant vanishes, allowing us to write the fourth order correlation in terms of the products of second order correlations:

$$
\begin{aligned}
i \frac{\partial \rho}{\partial \tau}+ & \beta_{1}\left(A^{2} \rho-B^{2} \rho\right)+i \beta_{2}\left(A^{3} \rho+B^{3} \rho\right) \\
= & 4 \Lambda_{1} \rho \sinh (M) \overline{a^{2}}(X, \tau)+i \Lambda_{2} \rho \cosh (M) \frac{\partial}{\partial X} \overline{a^{2}}(X, \tau) \\
& +i \Lambda_{2} \frac{\partial \rho}{\partial X} \cosh (M) \overline{a^{2}}(X, \tau)+2 i \Lambda_{2} \frac{\partial \rho}{\partial r} \sinh (M) \overline{a^{2}}(X, \tau) \\
& +2 i \Lambda_{3} \rho \cosh (M) \overline{a^{2}}(X, \tau)+\frac{2 \Lambda_{4}}{\pi} \sinh (M) \int_{-\infty}^{\infty} \frac{\partial \xi^{\prime}}{\xi^{\prime}-X} \frac{\partial}{\partial \xi^{\prime}} \overline{a^{2}}\left(\xi^{\prime}, \tau\right)
\end{aligned}
$$

where the operators $A, B, M$ are defined by

$$
A=\frac{1}{2} \frac{\partial}{\partial X}+\frac{\partial}{\partial r}, \quad B=\frac{1}{2} \frac{\partial}{\partial Y}-\frac{\partial}{\partial r}, \quad M=\frac{1}{2} r \frac{\partial}{\partial X}
$$


and $\overline{a^{2}}(X, \tau)$ is the ensemble averaged mean square amplitude given by

$$
\overline{a^{2}}(X, \tau)=\left\langle\zeta(X, \tau) \zeta^{*}(X, \tau)\right\rangle
$$

\section{Spectral transport equation}

The wave-envelope power spectral density $F(p, X, \tau)$ is defined by the Fourier transform of the two-point correlation function and is given by

$$
F(p, X, \tau)=\frac{1}{2 \pi} \int_{-\infty}^{\infty} e^{-i p r} \rho d r,
$$

where

$$
\rho=\left\langle\zeta\left(X+\frac{1}{2} r, \tau\right) \zeta^{*}\left(X-\frac{1}{2} r, \tau\right)\right\rangle .
$$

Taking the Fourier inversion of (15) we get

$$
\rho=\int_{-\infty}^{\infty} F(p, X, \tau) e^{i p r} d p
$$

from which on setting $r=0$ the following relation is obtained:

$$
\overline{a^{2}}(X, \tau)=\int_{-\infty}^{\infty} F(p, X, \tau) d p .
$$

Now taking the Fourier transform of (12) with respect to $r$, we get the following transport equation for $F$.

$$
\begin{aligned}
\frac{\partial F}{\partial \tau}+ & 2 \beta_{1} p \frac{\partial F}{\partial X}+\beta_{2}\left(\frac{1}{4} \frac{\partial^{3} F}{\partial X^{3}}-3 p^{2} \frac{\partial F}{\partial X}\right) \\
= & 4 \Lambda_{1} \sin (L) F \overline{a^{2}}+\Lambda_{2} \cos (L) F \frac{\partial \overline{a^{2}}}{\partial X} \\
& +\Lambda_{2} \cos (L) \frac{\partial F}{\partial X} \overline{a^{2}}-2 \Lambda_{2} \sin (L) p F \overline{a^{2}} \\
& +2 \Lambda_{3} \cos (L) F \frac{\partial \overline{a^{2}}}{\partial X}+\frac{2 \Lambda_{4}}{\pi} \sin (L) F \int_{-\infty}^{\infty} \frac{d \xi^{\prime}}{\xi^{\prime}-X} \frac{\partial}{\partial \xi^{\prime}} \overline{a^{2}}\left(\xi^{\prime}, \tau\right),
\end{aligned}
$$

where

$$
L=\frac{1}{2} \frac{\partial^{2}}{\partial X \partial p}
$$

and where $\partial / \partial X$ operates on $\overline{a^{2}}$ and $\partial / \partial p$ on $F$. 


\section{Stability analysis}

The nonlinear transport equation (19) has one basic solution

$$
F=F_{0}(p)
$$

which is independent of $X$ and $\tau$ and is the random counterpart of the uniform amplitude Stokes wavetrain of deterministic theory. To investigate the stability of this homogeneous solution to small amplitude oblique plane wave perturbation, we assume a perturbed solution of (19) in the form

$$
\begin{gathered}
F(p, X, \tau)=F_{0}(p)+F_{1}(p, X, \tau), \\
\overline{a^{2}}=\overline{a_{0}^{2}}+\overline{a_{1}^{2}}(X, \tau) .
\end{gathered}
$$

According to (18), $\overline{a_{0}^{2}}$ and $\overline{a_{1}^{2}}$ satisfy the relations

$$
\overline{a_{0}^{2}}=\int_{-\infty}^{\infty} F_{0}(p) d p, \quad \overline{a_{1}^{2}}=\int_{-\infty}^{\infty} F_{1}(p, X, \tau) d p .
$$

Substituting (22) and (23) in (19) and then linearizing we get

$$
\begin{aligned}
\frac{\partial F_{1}}{\partial \tau}+ & 2 \beta_{1} p \frac{\partial F_{1}}{\partial X}+\beta_{2}\left(\frac{1}{4} \frac{\partial^{3} F_{1}}{\partial X^{3}}-3 p^{3} \frac{\partial F_{1}}{\partial X}\right) \\
= & 4 \Lambda_{1} \sin (L) F_{0} \overline{a_{1}^{2}}+\Lambda_{2} \cos (L) F_{0} \frac{\partial \overline{a_{1}^{2}}}{\partial X} \\
& +\Lambda_{2} \cos (L) \frac{\partial F_{1}}{\partial X} \overline{a_{1}^{2}}-2 \Lambda_{2} \sin (L) p F_{0} \overline{a_{1}^{2}} \\
& +2 \Lambda_{3} \cos (L) F_{0} \frac{\partial \overline{a_{1}^{2}}}{\partial X}+\frac{2 \Lambda_{4}}{\pi} \sin (L) F_{0} \int_{-\infty}^{\infty} \frac{d \xi^{\prime}}{\xi^{\prime}-X} \frac{\partial}{\partial \xi^{\prime}} \overline{a_{1}^{2}}\left(\xi^{\prime}, \tau\right)
\end{aligned}
$$

Taking the Fourier transform of (25) with respect to $X$ defined according to

$$
\begin{aligned}
& \bar{F}_{1}=\int_{-\infty}^{\infty} F_{1}(X, \tau) e^{-i \ell X} d X \\
& \overline{A_{1}}=\int_{-\infty}^{\infty} \overline{a_{1}^{2}}(X, \tau) e^{-i \ell X} d X
\end{aligned}
$$

and then assuming $\tau$-dependence of $\bar{F}_{1}(\tau)$ and $\bar{A}_{1}(\tau)$ to be of the form $\exp (-i \Omega \tau)$, 
we get

$$
\begin{aligned}
-\Omega+ & 2 \beta_{1} p \ell-\beta_{2} \ell\left(\frac{1}{4} \ell^{2}-3 p^{2}\right) \bar{F}_{1} \\
= & 4 \Lambda_{1} \overline{A_{1}} \sinh \left(\frac{1}{2} \ell \frac{\partial}{\partial p}\right) F_{0}+\Lambda_{2} \ell \overline{A_{1}} \cosh \left(\frac{1}{2} \ell \frac{\partial}{\partial p}\right) F_{0} \\
& +\Lambda_{2} \ell \bar{F}_{1} \overline{a_{0}^{2}}-2 \Lambda_{2} \overline{A_{1}} \sinh \left(\frac{1}{2} \ell \frac{\partial}{\partial p}\right)\left(p F_{0}\right) \\
& +2 \Lambda_{3} \ell \overline{A_{1}} \cosh \left(\frac{1}{2} \ell \frac{\partial}{\partial p}\right) F_{0}-2 \Lambda_{4}|\ell| \overline{A_{1}} \sinh \left(\frac{1}{2} \ell \frac{\partial}{\partial p}\right) F_{0} .
\end{aligned}
$$

Similarly taking the Fourier transform of the second equation (24) we get

$$
\overline{A_{1}}=\int_{-\infty}^{\infty} \overline{F_{1}} d p
$$

Now in view of the relations (Alber [1])

$$
\begin{aligned}
\cosh \left(\frac{1}{2} \ell \frac{\partial}{\partial p}\right) F_{0} & =\frac{1}{2}\left[F_{0}\left(p+\frac{1}{2} \ell\right)+F_{0}\left(p-\frac{1}{2} \ell\right)\right], \\
\sinh \left(\frac{1}{2} \ell \frac{\partial}{\partial p}\right) F_{0}= & \frac{1}{2}\left[F_{0}\left(p+\frac{1}{2} \ell\right)-F_{0}\left(p-\frac{1}{2} \ell\right)\right], \\
\sinh \left(\frac{1}{2} \ell \frac{\partial}{\partial p}\right) p F_{0}= & \frac{1}{2} p\left[F_{0}\left(p+\frac{1}{2} \ell\right)-F_{0}\left(p-\frac{1}{2} \ell\right)\right] \\
& +\frac{\ell}{4}\left[F_{0}\left(p+\frac{1}{2} \ell\right)+F_{0}\left(p-\frac{1}{2} \ell\right)\right],
\end{aligned}
$$

(27) can be written as

$$
[-\Omega+f(p)] \bar{F}_{1}=\left[g(p) F_{0}\left(p-\frac{1}{2} \ell\right)+h(p) F_{0}\left(p+\frac{1}{2} \ell\right)\right] \overline{A_{1}},
$$

where

$$
\begin{aligned}
& f(p)=2 \beta_{1} p \ell-2 \beta_{2} \ell\left(\frac{1}{4} \ell^{2}-3 p^{2}\right)-\Lambda_{2} \ell \overline{a_{0}^{2}} \\
& g(p)=-2 \Lambda_{1}+\Lambda_{2} p+\Lambda_{3} \ell+\Lambda_{4}|\ell| \\
& h(p)=2 \Lambda_{1}-\Lambda_{2} p+\Lambda_{3} \ell-\Lambda_{4}|\ell| .
\end{aligned}
$$

From (30) we get

$$
\int_{-\infty}^{\infty} \bar{F}_{1} d p=\bar{A}_{1} \int_{-\infty}^{\infty} \frac{g(p) F_{0}\left(p-\frac{1}{2} \ell\right)+h(p) F_{0}\left(p+\frac{1}{2} \ell\right)}{-\Omega+f(p)} d p
$$


which in view of (28) gives the following dispersion relation determining $\Omega$ :

$$
\int_{-\infty}^{\infty} \frac{g(p) F_{0}\left(p-\frac{1}{2} \ell\right)+h(p) F_{0}\left(p+\frac{1}{2} \ell\right)}{-\Omega+f(p)} d p=1 .
$$

Now let

$$
\begin{aligned}
& f(p)=2 \beta_{1} p \ell+\epsilon f_{1}(p), \\
& g(p)=-2 \Lambda_{1}+\epsilon g_{1}(p), \\
& h(p)=2 \Lambda_{1}+\epsilon h_{1}(p),
\end{aligned}
$$

where $O(\epsilon)$ terms originate from the fourth order terms in the evolution equation (4) and are given by

$$
\begin{aligned}
& f_{1}(p)=-\beta_{2} \ell\left(\frac{1}{4} \ell^{2}-3 p^{2}\right)-\Lambda_{2} \ell \overline{a_{0}^{2}} \\
& g_{1}(p)=\Lambda_{2} p+\Lambda_{3} \ell+\Lambda_{4}|\ell| \\
& h_{1}(p)=-\Lambda_{2} p+\Lambda_{3} \ell-\Lambda_{4}|\ell|
\end{aligned}
$$

Substituting the expressions (34) for $f(p), g(p), h(p)$ in (33) and keeping terms up to order $\epsilon$, the equation determining $\Omega$ can be expressed as

$$
\begin{aligned}
2 \Lambda_{1} & \int_{-\infty}^{\infty} \frac{F_{0}\left(p+\frac{1}{2} \ell\right)-F_{0}\left(p-\frac{1}{2} \ell\right)}{\Omega-2 \beta_{1} \ell p} d p+1 \\
\quad= & 2 \epsilon \Lambda_{1} \int_{-\infty}^{\infty} \frac{f_{1}(p)\left[F_{0}\left(p-\frac{1}{2} \ell\right)-F_{0}\left(p+\frac{1}{2} \ell\right)\right]}{\left(\Omega-2 \beta_{1} \ell p\right)^{2}} d p \\
& -\epsilon \int_{-\infty}^{\infty} \frac{g_{1}(p) F_{0}\left(p-\frac{1}{2} \ell\right)+h_{1}(p) F_{0}\left(p-\frac{1}{2} \ell\right)}{\Omega-2 \beta_{1} \ell p} d p .
\end{aligned}
$$

For $F_{0}(p)$ we assume a Lorentz shape of spectrum in the form

$$
F_{0}(p)=\frac{\overline{a_{0}^{2}} \sigma}{\pi\left(p^{2}+\sigma^{2}\right)},
$$

where $\sigma$ is the width of the spectrum and $\overline{a_{0}^{2}}$ is the mean square wave steepness.

Now we shall first determine the roots of the dispersion relation (36) neglecting $O(\epsilon)$ terms, which is given in subsection-A. The roots of the same equation including order $\epsilon$ terms will be determined in following subsection-B. 
(A) Roots of the dispersion relation neglecting $O(\epsilon)$ terms Neglecting $O(\epsilon)$ terms the dispersion relation (36) becomes

$$
2 \Lambda_{1} \int_{-\infty}^{\infty} \frac{F_{0}\left(p+\frac{1}{2} \ell\right)-F_{0}\left(p-\frac{1}{2} \ell\right)}{\Omega-2 \beta_{1} \ell p} d p+1=0 .
$$

Setting $\Omega=\Omega_{r}+i \Omega_{i}$, where $\Omega_{r}$ and $\Omega_{i}$ are real and imaginary parts of $\Omega$, and using the property that $F_{0}(p)$ given by (37) is an even function of $p$, we can very easily prove that $\Omega_{r}=0$. So $\Omega$ is purely imaginary. Therefore replacing $\Omega$ by $i \Omega$, where $\Omega$ is real, and substituting $F_{0}$ given by (37) the dispersion relation (38) can be written as

$$
\begin{aligned}
\frac{2 \Lambda_{1} \overline{a_{0}^{2}} \sigma}{\pi} & \int_{-\infty}^{\infty} \frac{d p}{\left(p^{2}+\sigma^{2}\right)\left(i \Omega-\beta \ell^{2}+2 \beta \ell p\right)} \\
& -\frac{2 \Lambda_{1} \bar{a}_{0}^{2} \sigma}{\pi} \int_{-\infty}^{\infty} \frac{d p}{\left(p^{2}+\sigma^{2}\right)\left(i \Omega+\beta \ell^{2}+2 \beta \ell p\right)}+1=0,
\end{aligned}
$$

where we have set $\beta_{1}=-\beta$.

Since we are interested in the solution of the dispersion relation (39) for which the perturbation $F_{1}$ in $F$ grows with time, that is, for which there is instability, we must have $\Omega>0$. Keeping this in mind we perform the integrals appearing in (39) by contour integration. We thus get the following solution for $\Omega$.

$$
\Omega=2 \beta \ell\left[\left(\frac{\Lambda_{1} \overline{a_{0}^{2}}}{\beta}-\frac{\ell^{2}}{4}\right)^{1 / 2}-\sigma\right] \equiv \Omega_{i} .
$$

This will be the solution of (39) if $\Omega_{i}>0$.

(B) Roots of the dispersion relation including $O(\epsilon)$ terms The dispersion relation (36) including $O(\epsilon)$ terms can be put in the form

$$
\phi(\Omega)=\epsilon \psi(\Omega)
$$

where

$$
\phi(\Omega)=-2 \Lambda_{1} \int_{-\infty}^{\infty} \frac{F_{0}\left(p-\frac{1}{2} \ell\right)-F_{0}\left(p+\frac{1}{2} \ell\right)}{\Omega+2 \beta \ell p} d p+1
$$

and

$$
\begin{aligned}
\psi(\Omega)= & 2 \Lambda_{1} \int_{-\infty}^{\infty} \frac{f_{1}(p)\left[F_{0}\left(p-\frac{1}{2} \ell\right)-F_{0}\left(p+\frac{1}{2} \ell\right)\right]}{(\Omega+2 \beta \ell p)^{2}} d p \\
& -\int_{-\infty}^{\infty} \frac{g_{1}(p) F_{0}\left(p-\frac{1}{2} \ell\right)+h_{1}(p) F_{0}\left(p+\frac{1}{2} \ell\right)}{\Omega+2 \beta \ell p} d p .
\end{aligned}
$$


We have already seen in subsection-A that there exists a root of the equation

$$
\phi(\Omega)=0
$$

of the form

$$
\Omega=i \Omega_{i}, \quad\left(\Omega_{i}>0\right)
$$

So let the root of (41) be

$$
\Omega=i \Omega_{i}+\epsilon \Omega_{1}
$$

Substituting this in (41) we find that $\Omega_{1}$ in the lowest order is given by

$$
\Omega_{1}=\psi\left(i \Omega_{i}\right) / \phi^{\prime}\left(i \Omega_{i}\right)
$$

We easily find that

$$
\begin{aligned}
\psi\left(i \Omega_{i}\right) & =2 \Lambda_{1}\left(I_{1}-I_{2}\right)-\left(I_{3}+I_{4}\right), \\
\phi^{\prime}\left(i \Omega_{i}\right) & =\frac{2 \Lambda_{1} \overline{a_{0}^{2}} \sigma}{\pi}\left(I_{1}-I_{2}\right),
\end{aligned}
$$

where

$$
\begin{aligned}
& I_{1}=\int_{-\infty}^{\infty} \frac{d p}{\left(p^{2}+\sigma^{2}\right)\left(i \Omega_{i}+\beta \ell^{2}+2 \beta \ell p\right)^{2}} \\
& I_{2}=\int_{-\infty}^{\infty} \frac{d p}{\left(p^{2}+\sigma^{2}\right)\left(i \Omega_{i}-\beta \ell^{2}+2 \beta \ell p\right)^{2}} \\
& I_{3}=\frac{\overline{a_{0}^{2}} \sigma}{\pi} \int_{-\infty}^{\infty} \frac{\frac{1}{2} \Lambda_{2} \ell+\Lambda_{3} \ell+\Lambda_{4}|\ell|+\Lambda_{2} p}{\left(p^{2}+\sigma^{2}\right)\left(i \Omega_{i}+\beta \ell^{2}+2 \beta \ell p\right)^{2}} d p \\
& I_{4}=\frac{\overline{a_{0}^{2}} \sigma}{\pi} \int_{-\infty}^{\infty} \frac{\frac{1}{2} \Lambda_{2} \ell+\Lambda_{3} \ell-\Lambda_{4}|\ell|-\Lambda_{2} p}{\left(p^{2}+\sigma^{2}\right)\left(i \Omega_{i}-\beta \ell^{2}+2 \beta \ell p\right)} d p
\end{aligned}
$$

Contour integration now gives $\Omega_{1}$; we get

$$
\operatorname{Im}\left(\Omega_{1}\right)=-\frac{\Lambda_{4}|\ell|}{4 \Lambda_{1}} \frac{\left(\Omega_{i}+2 \beta \ell \sigma\right)^{2}+\beta^{2} \ell^{4}}{\left(\Omega_{i}+2 \beta \ell \sigma\right)}
$$

Since the growth rate of instability $\gamma_{1}$ is equal to $\operatorname{Im}(\Omega)$, from (46) and (51) we get

$$
\gamma_{1}=\Omega_{i}-\frac{\Lambda_{4}|\ell|}{4 \Lambda_{1}} \frac{\left(\Omega_{i}+2 \beta \ell \sigma\right)^{2}+\beta^{2} \ell^{4}}{\left(\Omega_{i}+2 \beta \ell \sigma\right)},
$$


where $\Omega_{i}$ is given by (40). The second term in (52) is the contribution from the fourth order term in the nonlinear evolution equation, and this term produces a decrease in the growth rate of instability. $\Omega_{i}$ is the growth rate of the instability obtained from the third order nonlinear evolution equation and this decreases with the increase in bandwidth $\sigma$.

The deterministic growth rate of the instability obtained in our previous paper [2] is recovered from (52) by setting $\sigma=0$. Since by setting $\sigma=0$ in (52) we get

$$
\gamma_{1}=\Omega_{i 0}-\frac{\Lambda_{4} \overline{a_{0}^{2}}|\ell| \ell^{2} \beta}{\Omega_{i 0}}
$$

where

$$
\Omega_{i 0}=\beta^{1 / 2} \ell \sqrt{4 \Lambda_{1} \overline{a_{0}^{2}}-\beta \ell^{2}} .
$$

From equation (45) in [2] we get the following expression for the deterministic growth rate of the instability:

$$
\gamma_{1}=\Omega_{i 0}\left(1-\frac{2 \Lambda_{4} a_{0}^{2}|\ell| \ell^{2} \beta}{\Omega_{i 0}^{2}}\right)^{1 / 2}=\Omega_{i 0}-\frac{\Lambda_{4} a_{0}^{2}|\ell| \ell^{2} \beta}{\Omega_{i 0}}
$$

(expanding binomially and then neglecting higher order small terms). Equation (55) is same as (53) if $\overline{a_{0}^{2}}$ is identified with $a_{0}^{2}$.

Introducing the effective modulation wave number $\bar{\ell}$ and the effective bandwidth parameter $\bar{\sigma}$ by

$$
\bar{\ell}^{2}=\frac{\ell^{2}}{\overline{a_{0}^{2}}}, \quad \bar{\sigma}^{2}=\frac{\sigma^{2}}{\overline{a_{0}^{2}}},
$$

the effective growth rate of the instability $\bar{\gamma}=\gamma_{1} / \overline{a_{0}^{2}}$ can be expressed as follows according to (52):

$$
\bar{\gamma}=\bar{\Omega}_{i}-\frac{\Lambda_{4}|\bar{\ell}|}{4 \Lambda_{1}} \bar{a}_{0} \frac{\left(\bar{\Omega}_{i}+2 \beta \bar{\ell} \bar{\sigma}\right)^{2}+\beta^{2} \bar{\ell}^{4}}{\left(\bar{\Omega}_{i}+2 \beta \bar{\ell} \bar{\sigma}\right)}
$$

where

$$
\bar{\Omega}_{i}=2 \beta \bar{\ell}\left(\sqrt{\frac{\Lambda_{1}}{\beta}-\frac{\bar{\ell}^{2}}{4}}-\bar{\sigma}\right), \quad \bar{a}_{0}=\left(\overline{a_{0}^{2}}\right)^{1 / 2}
$$




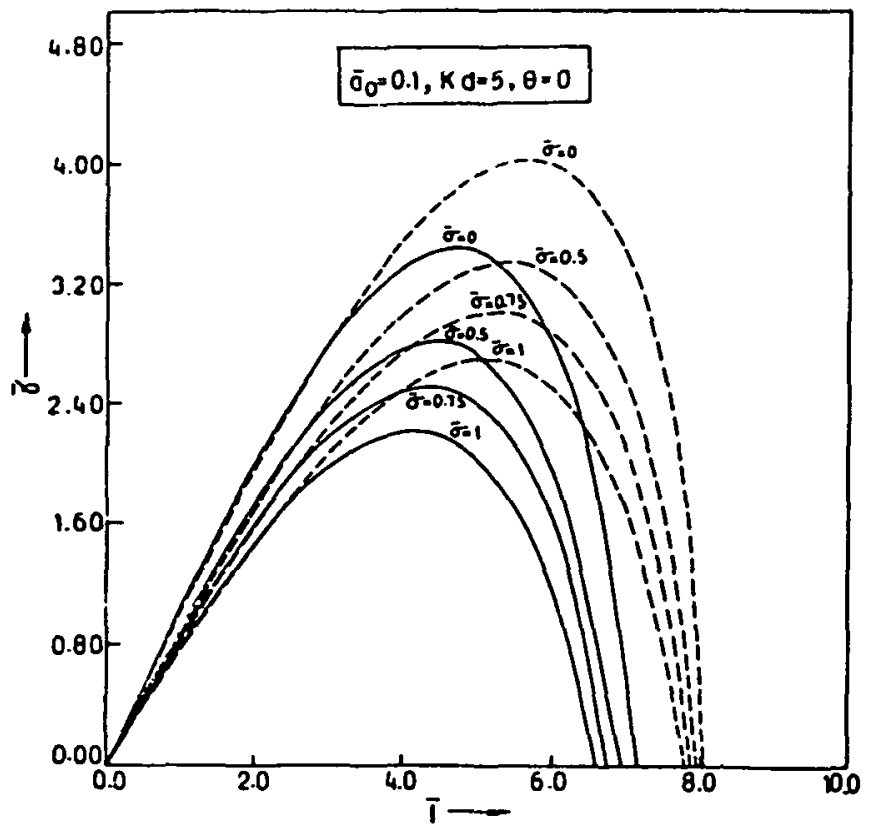

(a)

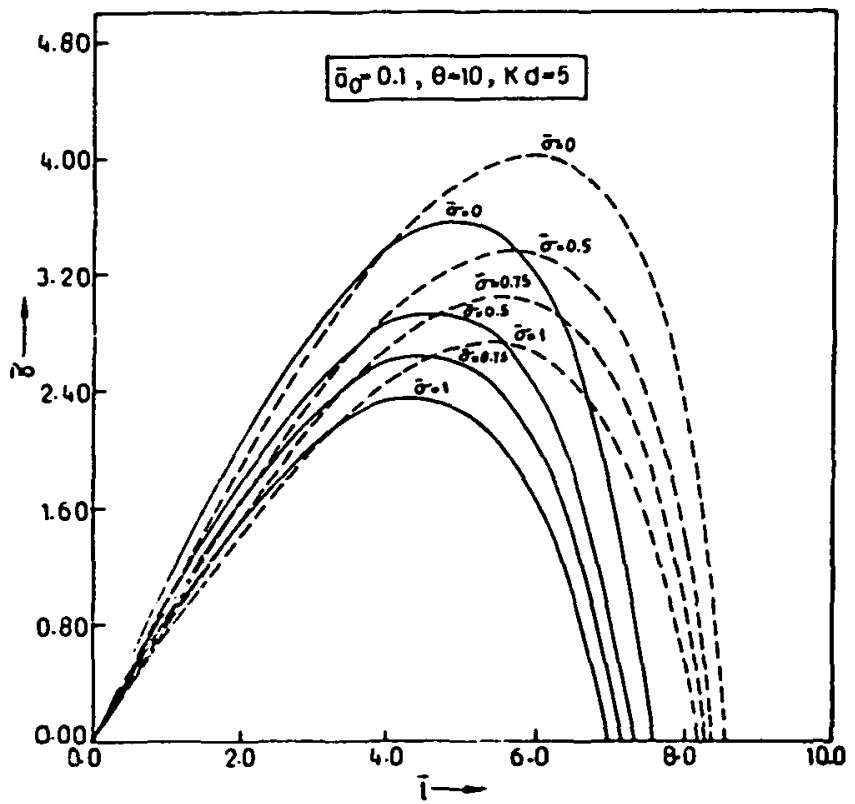

(b) 


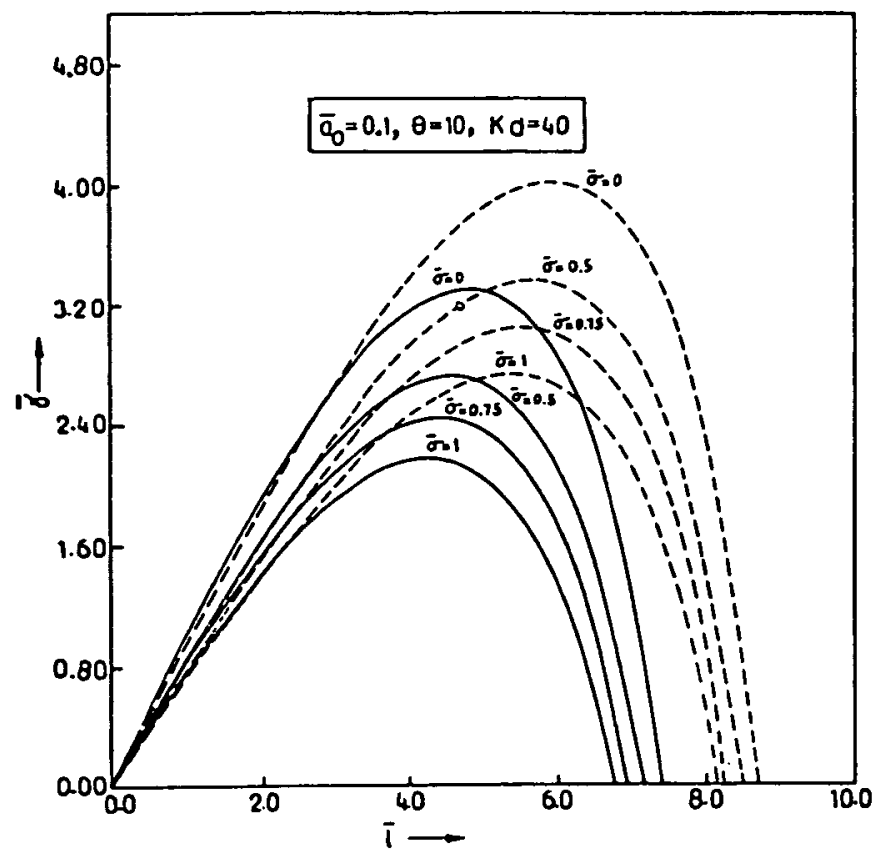

(c)

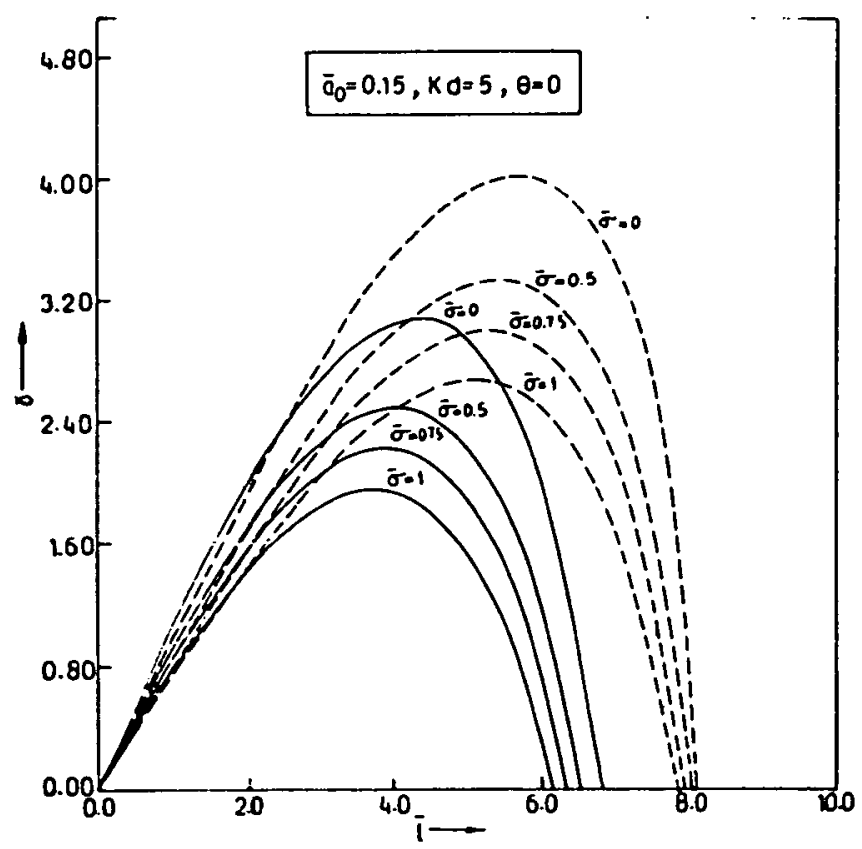

(d) 


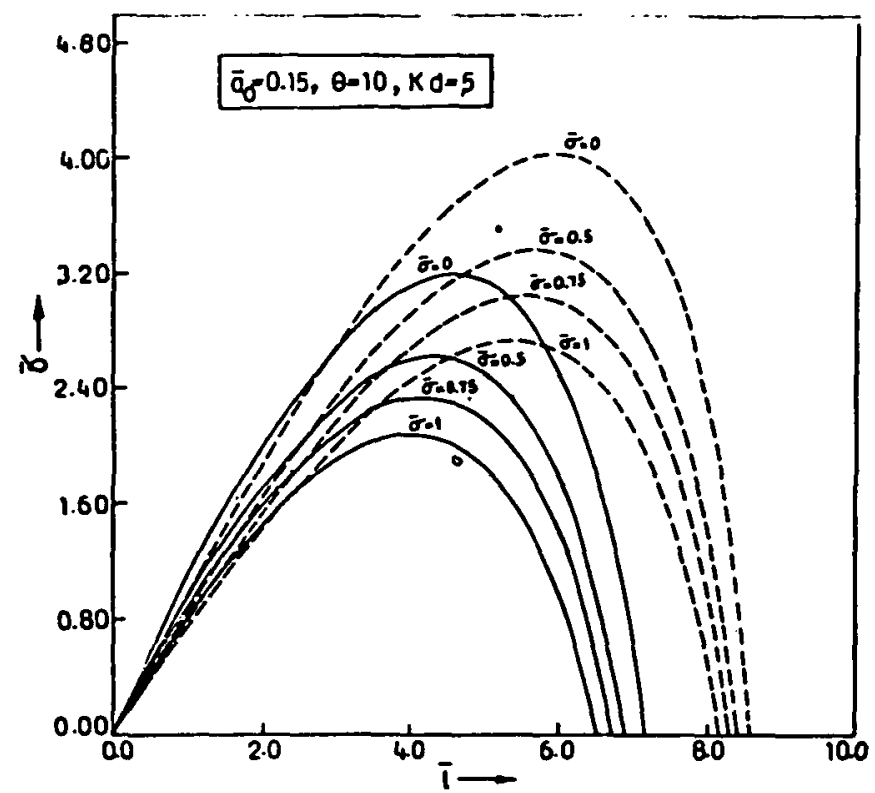

(e)

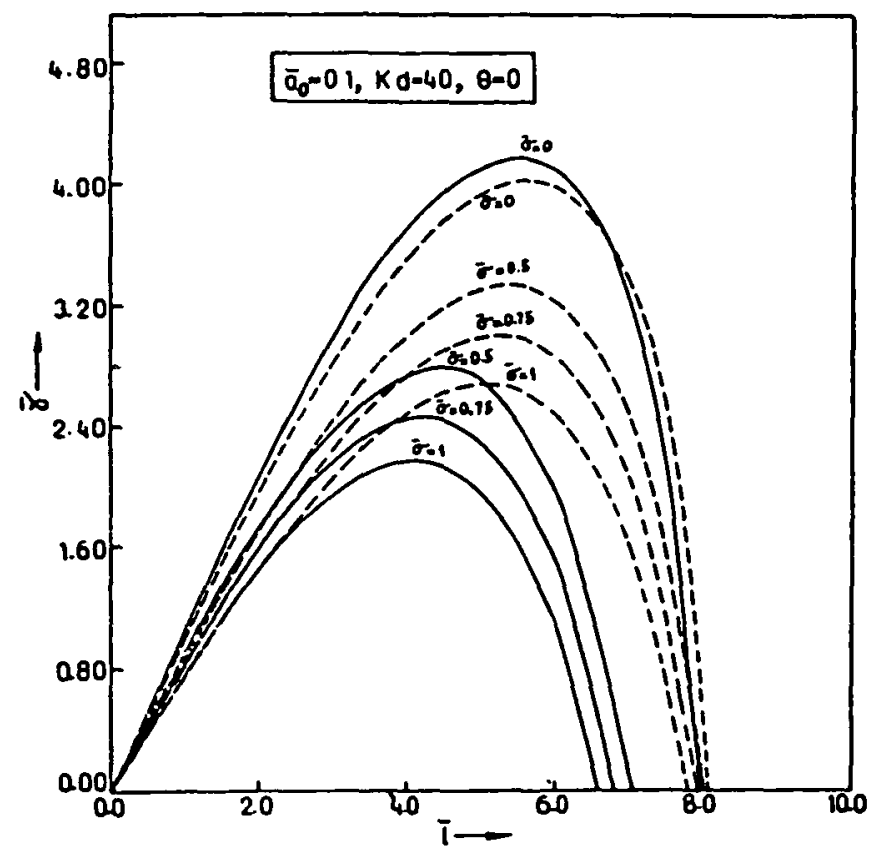

(f)

FIGURE 1. Effective growth rate of instability $\bar{\gamma}$ against effective modulation wave number $\bar{\ell} ;-$ curves represent third order results. 
In Figures 1(a)-1(f) $\bar{\gamma}$ has been plotted against the effective modulation wavenumber $\bar{\ell}$ for some different values of effective bandwidth parameter $\bar{\sigma}$ and also for some different values of dimensionless thermocline depth $k d$, perturbation angle $\theta$ and rootmean square wave steepness $\bar{a}_{0}$. The third order growth rate of the instability $\bar{\Omega}_{i}$ is shown by broken lines. The curve for $\bar{\sigma}=0$ gives the deterministic growth rate of the instability.

From the graphs it is clear that there is no instability if the slope of the growth rate of the instability curve is negative at $\bar{\ell}=0$. This gives the following condition for stability

$$
\left(\frac{d \gamma_{1}}{d \ell}\right)_{\ell=0}<0
$$

Evaluating the derivative $\left(d \gamma_{1} / d \ell\right)_{\ell=0}$ from the expression for $\gamma_{1}$ given by (52) we get the following condition for stability:

$$
\sigma>\left(\frac{\Lambda_{1} \overline{a_{0}^{2}}}{\beta}\right)^{1 / 2} .
$$

This implies that there is stability, if band-width exceeds a certain critical value.

The $\bar{\gamma}=0$ curves given by (57) for fixed values of $\theta, k d, \bar{a}_{0}$ separate the $\bar{\ell} \bar{\sigma}-$ plane into stable-unstable regions. Such curves have been drawn in Figures 2(a), 2(b).

\section{Conclusion}

The effect of randomness of the stability of deep water surface gravity waves in the presence of a thin thermocline has been investigated. Following Alber [1] a spectral transport equation for narrow band surface gravity wave trains, slowly varying with space co-ordinates and time, is derived from the nonlinear evolution equation obtained by Bhattacharyya and Das [2], which includes the existence of a thin thermocline. Stability analysis is then made from this transport equation for an initially homogeneous Lorentz shape of spectrum to a small long wavelength perturbation. An expression for the growth rate of the instability has been obtained, which coincides with the deterministic growth rate of instability when the band-width vanishes. It is found that the growth rate of the instability decreases with the increase of spectral width. The fourth order term in the evolution equation, which is responsible for the wave-induced mean flow, produces a decrease in the growth rate of the instability. The instability vanishes if the spectral width increases beyond a certain critical value. The growth rate of the instability against the wave number of the perturbation has been shown graphically for some different values of the spectral width, thermocline depth, 


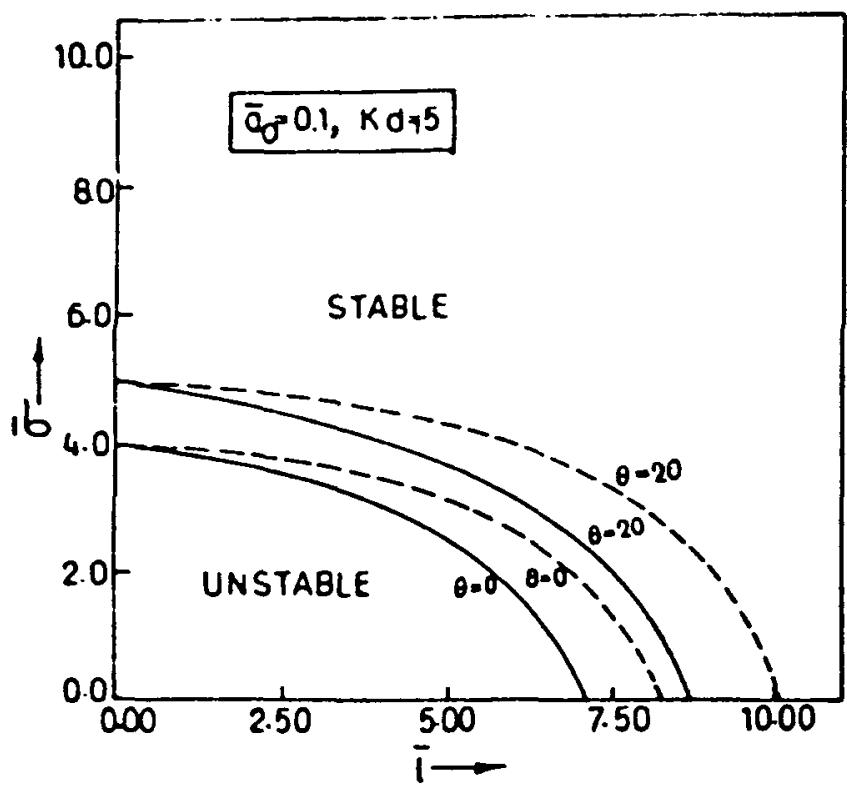

(a)

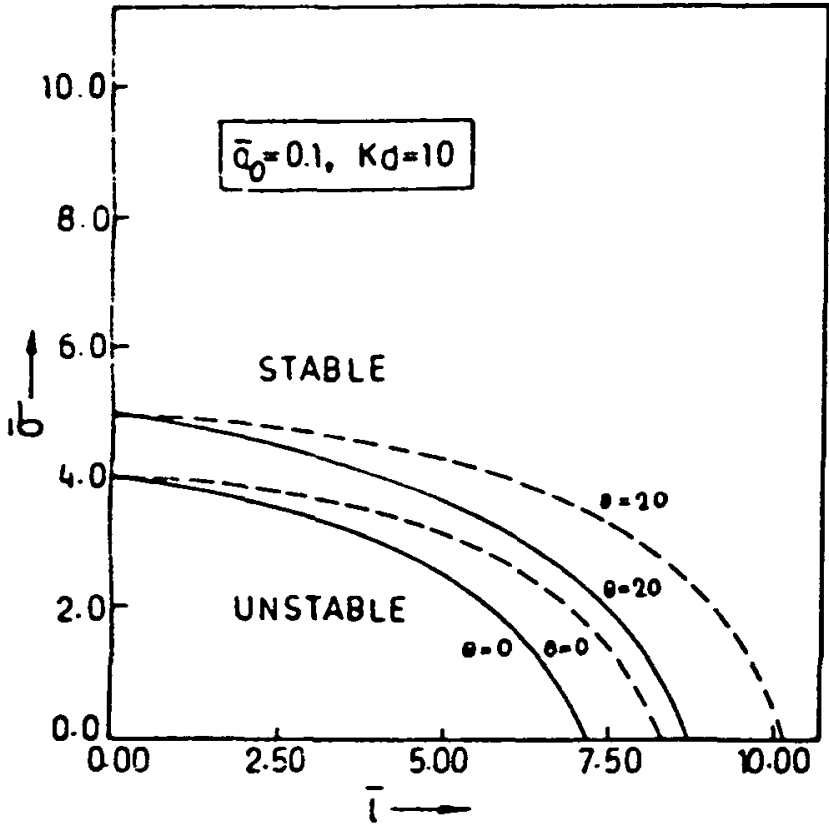

(b)

FIGURE 2. Curves separating stable-unstable regions in $\bar{\ell} \bar{\sigma}-\longrightarrow$ curves represent third order results. 
angle of perturbation and mean square wave steepness. Stable-unstable regions in the (perturbation wave number)-(spectral width) plane are also shown graphically.

\section{References}

[1] I. E. Alber, "The effects of randomness on the stability of two-dimensional wavetrains", Proc. $R$. Soc. Lond. A363 (1978) 525-546.

[2] S. Bhattacharyya and K. P. Das, "Fourth order non-linear evolution equations for surface gravity waves in the presence of a thin thermocline", J. Austral. Math. Soc. Ser. B 39 (1997), to be published.

[3] D. R. Srawford, P. G. Saffman and H. C. Yuen, "Evolution of a random inhomogeneous field of nonlinear deep-water gravity waves", Wave Motion 2 (1980) 1-16.

[4] K. B. Dysthe, "Note on a modification to the nonlinear Schrödinger equation for application to deep water waves", Proc. R. Soc. Lond. A369 (1979) 105-115.

[5] M. S. Longuet-Higgins, "The instabilities of gravity waves of finite amplitude in deep water I. Super harmonics", Proc. R. Soc. Lond. A360 (1978) 471-488.

[6] M. S. Longuet-Higgins, "The instabilities of gravity waves of finite amplitude in deep water II. Subharmonics", Proc. R. Soc. Lond. A360 (1978) 489-505.

[7] V. E. Zakharov, "Stability of periodic waves of finite amplitude on the surface of deep fluid", $J$. Appl. Meh. Tech. Phys. 2 (1968) 190-194. 